\title{
Mesure et Analyse de l'Efficacité des Etablissements de la Formation Professionnelle en Tunisie
}

\author{
Ezzeddine Mosbah, (ONQ\&M, MoET, Tunisie) \\ Mohamed S. Bachta, (INAT, Tunisie)
}

\begin{abstract}
Résumé
Le présent travail a pour objectif de mesurer et d'analyser l'efficacité des établissements de la formation professionnelle en Tunisie. Pour cela, une méthodologie en deux étapes a été utilisée. La première étape concerne l'estimation de l'efficacité technique par le modèle BCC (1984). La deuxième étape estime l'efficacité allocative en utilisant les modèles des coûts d'ombre: Kumbhakar (1996) et Balk (1997). Les principaux résultats montrent, d'une part, que ces établissements peuvent être classés, par niveau d'efficacité technique, en trois groupes. Le groupe hautement efficace, dont les scores dépassent 0.8 , renferme $20 \%$ des établissements. Le groupe moyennement efficace, dont les scores sont entre 0.5 et 0.79 , renferme $46 \%$. Enfin, le groupe faiblement efficace, avec des scores inferieurs à 0.5 , contient le reste des centres. D'autre part, les résultats montrent que les centres sont allocativement efficaces $(>0.8)$.
\end{abstract}

\begin{abstract}
:
This paper aims to measure and analyze efficiency of public training establishments in Tunisia. For that, a two-stage methodology is used. The first stage estimates technical efficiency scores using the BCC model (1984). The second, based on results of the first stage, estimates allocative ones using shadow cost models: Kumbhakar (1996) and Balk (1997). The main results provide, on the one hand, that establishments could be classified, by technical efficiency level, into three groups. The most efficient group, with scores higher than 0.8 , includes $20 \%$ of establishments. The middle efficient group, with scores between 0.5 and 0.79 , represents $46 \%$. Finally, the rest (34\%) represent a lower efficient group with scores under 0.5 . However, results show that centers are allocatively efficient $(>0.8)$.
\end{abstract}

\section{INTRODUCTION}

En Tunisie, la productivité globale des facteurs parait le principal stimulant de toute croissance. Pour cela, sa part va être renforcée pour atteindre les 50\% à l'horizon 2016 au lieu de 41\% actuellement (Ministère du Développement Economique et de la Coopération Internationale [MDECI], 2006, p.51). Ceci se réalisera principalement par la valorisation des ressources humaines destinées à répondre 
aux besoins de l'économie.

De ce fait, la Tunisie a lancé un programme de mise à niveau de la formation professionnelle (MANFORM) préparant un environnement favorable à une meilleure formation des ressources humaines et favorisant un changement fondamental des structures du dispositif visant l'efficacité de ses établissements. Ainsi, la période du $9^{\text {ème }}$ plan (1997-2001) a constitué la première phase de réalisation du programme, mobilisant environ 150 MDT [Million de Dinars Tunisiens] (Ministère de l'Education et de la Formation [MEF], 2001, p.»n.d»). Cependant, les avantages et les inconvénients du nouvel environnement en apparition, constituent le fond fort d'une question couramment posée: Dans quelle mesure le nouvel environnement incite-t-il l'efficacité des établissements de formation?

Dans ce sens, notre travail évaluera, à mis parcours du Programme, l'efficacité économique des établissements publics de formation répartis en offre géographique (Morlaix, 2001, p.4) bien déterminée sur le territoire Tunisien. C'est-à-dire, il s'agit de se demander à quel point ces établissements pourraientils préserver leurs performances techniques et allocatives en s'interrogeant avec Psaccharopoulos et Woodhall (1988) sur l'utilisation optimale des ressources tout en rejoignant De Ketele (1989) dans sa quête des meilleurs coûts de formation (cité dans Sall, 1996, p110). Plus précisément, notre travail abordera l'efficacité-coût (cost efficiency) des établissements de formation dont le concept est relatif à l'ampleur d'ajustement des coûts observés aux coûts minimums, et dont l'appréciation est relative au produit de ses composantes: technique et allocative. Le fait que le coût observé englobe le coût minimum, le coût dû à l'inefficacité technique et le coût dû à l'inefficacité allocative, il semble que l'efficacité technique dépend du choix et d'allocation des ressources utilisées dans le processus de formation par rapport à leur niveau optimal et que l'efficacité allocative est liée à l'ajustement des prix de ces ressources à leurs prix du marché.

Pour effectuer ce travail, la première partie présentera le statut décisionnel des établissements publics de formation. La deuxième s'intéressera à la méthodologie adoptée. Quand à la troisième partie, elle montrera l'étude empirique et les principaux résultats obtenus. Enfin, la quatrième partie conclura le travail.

\section{Les établissements de formation sont des unités de prise de décision}

Dans le cadre d'analyse fournie par l'économie de l'éducation, le processus de formation est perçu plus compliqué qu'un processus de production ordinaire. Il est, en effet, identifiable comme une transformation de la situation mentale de l'individu partant des connaissances générales (mathématiques, sciences, etc.) et aboutissant aux compétences professionnelles (savoir, savoir-faire et savoir-être). Dans ce sens, Psaccharopoulos et Woodhall (1988) ont suggéré que la fonction de production est peu perceptible pour la formation à cause de sa spécificité immatérielle et de son nombre de facteurs élevé (Cité dans Sall, 1996, p.108). 
Cependant, la théorie des firmes définit le processus de production comme l'ensemble des tâches successives permettant de transformer les facteurs en produits suivant une technologie et une organisation données. L'emprunt de cette définition et sa projection sur le processus de formation est possible, tout en respectant les spécificités immatérielles de ses facteurs et de ses produits (Mosbah, 2007). En plus, cette suggestion permet de simplifier le système en quelques facteurs représentatifs sans changer la structure générale de ce processus.

Dans le cas idéal, le processus de formation consiste à une mobilisation d'un nombre limité de ressources (postes de formation, personnel, programmes de formation) en faveur des individus éligibles suivant les différents modes (résidentiel, en alternance et/ou en apprentissage). Les produits obtenus sont les diplômés qui seront soit avantagés par le marché de l'emploi en favorisant leurs insertions soit sanctionnés en retardant leurs placements. Ce rôle d'arbitrage par le marché se joue en se référant aux exigences des entreprises et en attribuant une valeur à chaque diplômé.

A cette fonction de production des qualifications s'ajoute la responsabilité des directeurs de centres en matière d'allocation des ressources, du management, de contrôle qualité, de développement des relations de partenariat entreprise-centre. Ceux-ci mettent en évidence que les établissements publics de formation peuvent être rapprochés aux unités de décision économique ordinaire ayant, selon Morlaix (2000) des pratiques variées (cité dans Morlaix, 2001, p.6) et que les directeurs sont des agents rationnels dont le comportement est la minimisation des coûts de formation (Mosbah, 2007).

Dans la pratique, ces décideurs se heurtent à plusieurs problèmes. Premièrement, la difficulté de la préparation rigoureuse des programmes dûe à la capacité limitée des entreprises à exprimer leurs besoins en qualifications. Deuxièmement, la difficulté d'opposition au bon remplissage de la capacité et au plein emploi du personnel s'explique par la faible capacité des établissements à établir des relations partenariales parfaites avec les entreprises, le libre choix des individus éligibles à la formation (secteurs, régions, etc.), outre l'indisponibilité et de l'immobilité du personnel. Troisièmement, citons l'insertion retardée des diplômés dégénérant des fluctuations du marché de l'emploi et des caractéristiques des individus eux-mêmes.

Ces problèmes s'imposent de façons différentes aux établissements provoquant, selon Michaut (2000), des conditions de formation sensiblement variées (cité dans Morlaix, 2001, p.5), et traduisant une rationalité limitée des manageurs. Celle-ci se répercute mal sur la réalisation des objectifs dressés à la formation en mettant en cause l'efficacité de ces établissements.

\section{Approche méthodologique et techniques d'estimation de l'efficacité}

Selon la théorie néoclassique, les agents de production sont rationnels lorsqu'ils 
recourent soit à une maximisation du profit soit à une minimisation du coût. Le dernier choix, indiquant le sens d'orientation vers la réduction des inputs (OI), implique que le manageur procède par l'ajustement du coût observé au coût minimum.

Cette opération peut être effectuée en deux étapes successives. La première étape consiste à produire des qualifications avec des quantités réduites de facteurs par comparaison à des quantités utilisées auparavant. Cela veut dire que, pour un niveau donné de production, le manageur peut réduire au minimum possible le niveau d'un ou de plusieurs facteurs sans augmenter le niveau des autres. On peut dire encore que le décideur a évité ce que Samuelson (1965) appelle gaspillage des ressources. Donc, si cela est pratiquement possible, l'ancienne technique de production est jugée techniquement inefficace alors que la nouvelle l'est. Pour un ensemble d'établissements utilisant des différentes quantités de facteurs pour produire le même niveau de production, ceux qui utilisent les quantités minimales sont techniquement efficaces, quant aux autres ils peuvent atteindre la frontière d'efficacité technique lorsqu'ils procèdent par une réduction des unités de facteurs en excès.

La deuxième étape consiste à ajuster les prix des facteurs jusqu'aux niveaux obtenus sur le marché. Dans la réalité, la transparence du marché des inputs n'est pas parfaite causant des risques et des incertitudes qui influencent les décisions des manageurs. Ces derniers deviennent satisfaits pour procurer des facteurs avec des prix non observés encore appelés des prix d'ombre $\left(\mathrm{w}^{*}=\mathrm{r} . \mathrm{w}\right)$ supérieurs à ceux du marché (w). Ainsi, toute distance observée entre le coût minimum et le coût d'ombre (résultant des prix d'ombre) est à l'origine d'une inefficacité allocative (ou inefficacité prix).

Les deux étapes précédentes s'associent pour donner l'efficacité économique, encore appelée efficacité-coût (EC), obtenue à partir du produit des deux composantes technique (ET) et allocative (EA).

\section{Estimation de l'efficacité technique globale (ETG) et ses composantes pure (ETP) et d'échelle (ECH) par le modèle BCC(1984)}

Rappelons tout d'abord que sur la base des travaux de Debreu (1951) et Farrel (1957), Charnes, Cooper et Rhodes (1978) ont estimé l'efficacité technique à partir d'un enveloppement de données observées sur $\mathrm{n}$ unités de prise de décision $\mathrm{DMU}_{\mathrm{j}}$ $(j=1, \ldots, n)$. Chaque unité utilise $m$ facteurs $\left(x_{i}, i=1, \ldots, m\right)$ pour produire s produits $\left(\mathrm{y}_{\mathrm{r}}, \mathrm{r}=1, \ldots, \mathrm{s}\right)$. Le modèle développé, appelé CCR (1978), ne s'interprète que dans les conditions de rendements d'échelle constants.

L'interprétation élargie de ce modèle dans le cas des rendements d'échelle variables a dicté à Banker, Charnes et Cooper (1984) d'introduire les aspects relatifs à la convexité de la technologie. En effet, le modèle BCC (1984) a ajouté une contrainte portée sur le poids $(\mathrm{lj})$ attribué à chacune des $\mathrm{DMU}_{\mathrm{j}}$ déjà présente dans 
l'enveloppement de données ${ }^{1}$.

Cette contrainte, et selon la valeur donnée à la somme des poids, confère au modèle BCC(1984) deux formes. Le premier cas suppose que $\sum \lambda_{j}=1$ donne la version des rendements d'échelle constants (REC) du modèle $\mathrm{BCC}^{j}(1984)$, estimant l'ETG, similaire à la forme ordinaire du modèle CCR(1978). Le deuxième cas comprend deux propositions, soit $\sum \lambda_{j} \mathrm{f} 1$ indiquant ainsi des rendements d'échelle décroissants (RED), soit $\sum \lambda_{j} f 1^{j}$ reflétant les rendements d'échelle croissants (RECr), pour aboutir à l'id́dentification de la version des rendements d'échelle variables (REV) du modèle BCC (1984), qui est différente de celle de CCR (1978).

La résolution du modèle BCC (1984) pour l'unité de décision (k) donne la solution optimale $(\mathrm{qk}, \mathrm{j})$, où $\mathrm{qk} \hat{\mathrm{I}}[0 \mathrm{l}]$ et $\mathrm{lj}$ positif et quelconque. Les coefficients (qk) obtenus par l'estimation de la forme REC s'interprètent en termes de scores de l'efficacité technique globale (ETG). Par contre, les résultats (qk) obtenus par l'estimation de la forme REV s'interprètent en termes de scores de l'efficacité technique (ETP). Les scores de l'efficacité d'échelle ECH sont déduits à partir du rapport entre l'ETG et l'ETP.

\section{Estimation de l'efficacité allocative (EA) par le modèle des coûts d'ombre}

Cette étape consiste à déduire les scores de l'efficacité allocative (EA) à partir de l'estimation du modèle des coûts d'ombre. Rappelons tout d'abord que la théorie des coûts ordinaire suggère que les coûts observés $\left(\mathrm{C}^{\mathrm{ob}}\right)$ d'une unité de décision orientée vers la réduction des inputs sont égaux au produit des coûts minimums $\left(\mathrm{C}^{\circ}\right)$ et les coûts dûs à l'inefficacité technique (IT) outre ceux dûs à l'inefficacité allocative (IA).

Dans le même sens, la théorie des coûts d'ombre suggère que les décideurs, dans les conditions de l'information imparfaite, minimisent les coûts d'ombre $\left(C^{*}\right)$ au lieu des coûts minimums $\left(C^{0}\right)$. Kumbhakar (1996) stipule qu'il est possible d'écrire les coûts observés $\left(\mathrm{C}^{\mathrm{ob}}\right)$ comme des coûts d'ombre $\left(\mathrm{c}^{*}\right)$ majorés par les coûts dûs à l'inefficacité technique (IT) et les coûts dûs aux ajustements (Aj). La déduction des parts des coûts des facteurs $\left(S_{i}, i=1, \ldots, m-1\right)$ à partir de la forme translog, $\operatorname{LnC}^{*}\left(\mathrm{w}^{*}, \mathrm{y}\right)$, identifiée par Atkinson et Cornwell (1994) (cité dans Maeitta, 2000, p.482) permet d'obtenir le modèle des coûts d'ombre d'Atkinson, Cornwell et Kumbhaker (ACK) ${ }^{2}$.

Cependant, Balk (1997) suppose, en utilisant les suggestions de Mensah (1994) que les coûts totaux observés soient égaux aux coûts d'ombre totaux, $\mathrm{w}^{*} \mathrm{x}$ =wx (cité dans Maeitta, 2002, p.4). cela veut dire que les coûts d'ajustement (Aj) sont nuls, c'est-à-dire $\ln (A j)=0$. Par conséquent, le modèle ACK se simplifie en une forme particulière de Mensah et Balk $(\mathrm{MB})^{3}$. L'efficacité allocative sera déduite à partir de la forme développée d'ACK et MB.

L'estimation du modèle nécessite la prise des scores de l'ETG obtenus de la première étape comme données et l'identification des distorsions des prix des 
facteurs. Telle que citée par Maeitta (2000, p.484), la distorsion a été identifiée dans un cas par les caractéristiques des manageurs: l'âge, le niveau de l'éducation, et l'expérience professionnelle (Kumbhakar, 1996). Dans l'autre cas, cette distorsion a été décrite simultanément par le temps et les caractéristiques des firmes ellesmêmes (Balk \& Van Leeuman, 1997; Atkinson \& Cornwell, 1998)

Quand à notre spécification, elle a retenu les caractéristiques des établissements publics comme sources de distorsion tels que le niveau et le mode de formation adoptée, (Dij), la région de localisation, (Drj), et le secteur de formation, (Dsj). Ces caractéristiques ont respectivement les effets K(i), Cr et Cs. La distorsion sera introduite au modèle sous forme de ratio ${ }^{4}$.

Les variables retenues comme facteurs sont: la capacité de formation de l'établissement (j) en nombre de postes alloués pour réaliser un cycle de formation calculé par la double somme sur le nombre d'année de formation et le niveau de formation du produit de la capacité de formation annuelle et l'effectif des stagiaires. Puis, le facteur Travail qui est le volume d'homme/mois consacré à l'exercice du cycle de formation. Il est obtenu de la même façon sur le produit du volume annuel du travail et l'effectif des stagiaires.

Les variables liées au nombre d'individus inscrits, à un cycle de formation défini par le mode et le niveau de formation, sont en nombre de cinq correspondants à deux niveaux de formation et à trois modes. Pour chaque catégorie, on compte le nombre d'inscrits au début du cycle de formation $\left(\mathrm{NS}_{\mathrm{th}}\right)$.

La variable output pour un établissement de formation (j) est représentée par la valeur des diplômés sur le marché de l'emploi. Cette valeur se mesure en termes d'effectif des diplômés doublement pondéré, par le niveau et la situation sur le marché de l'emploi. La première pondération, retenant le nombre d'heures de formation, suppose qu'un BTP (Brevet de Technicien Professionnel), ou plus, représente deux unités de qualifications alors qu'une CAP (Certificat d'Aptitude Professionnelle) est seulement d'une unité. Ici, la mesure de l'efficacité de la formation se fait vis-à-vis du niveau de formation (niveau du diplôme obtenu) CAP, BTP.

La deuxième pondération s'obtient par l'application de la loi Rayleigh, sur les segments de périodes de 6 mois à partir de la sortie de l'établissement durant 3 ans. Cette loi attribue à ces périodes les coefficients: 1 pour la période allant de 0 à 6 mois, 0.64 pour 7-12 mois, 0.34 pour $13-18$ mois, 0.18 pour 19-24 mois, 0.09 pour 25-30 mois et 0.03 pour 31-36 mois.

Pour chaque facteur retenu et précédemment décrit, se définit un prix tels que le coût d'usage de la capacité, le salaire mensuel moyen du personnel, le coût moyen d'élaboration des programmes. Enfin, les coûts moyens de formation des individus par mode et niveau de formation ${ }^{5}$ calculés à partir d'une agrégation des dépenses en matériel de formation, en management, en électricité, etc. et ce pour chaque catégorie d'individus (par niveau et mode de formation). 


\section{ETUDE EMPIRIQUE}

L'étude empirique a été portée sur 60 établissements publics de la formation diplomante qui ont produit des qualifications au cours de l'année 2002. Ces établissements sont des centres sectoriels (CSF) et des centres de formation et d'apprentissage (CFA) ayant fonctionné au cours des deux années successives 2000 et 2001 .

Les données correspondantes aux activités de formation au niveau des centres sont issues des directions compétentes de l'Agence Tunisienne de la Formation Professionnelle (ATFP). Quant aux informations relatives à l'insertion des diplômés sur le marché de l'emploi, elles sont collectées en moyennant une enquête auprès de $10 \%$ (environ 1000 individus) du total des diplômés.

L'estimation des modèles a pris en compte les spécificités de la fonction des coûts d'ombre qui est monotone et homogène de degré 1 et symétrique et convexe sur les prix d'ombre des facteurs $\left(\mathrm{w}_{\mathrm{i}}{ }^{*}\right)$ et elle est croissante sur les produits.

\section{Analyse des résultats de l'estimation du modèle BCC(1984): l'ETG, l'ETP et l'ECH}

Les résultats de l'estimation (Annexes, tableau 1, $1^{\text {ère }}$ étape) de la forme REC du modèle BCC (1984) montrent que les établissements ont des performances techniques différentes. En effet, les scores de l'ETG varient entre 0.158 à 1 permettant la classification des établissements en 3 groupes différents. Le groupe (1) se compose de $25 \%$ des centres hautement efficaces dont les scores dépassent 0.807 . Ensuite, le groupe (2) renferme $43.3 \%$ des centres moyennement efficaces ayant les scores variant entre 0.512 et 0.761 . Enfin, le groupe (3) rassemble la part restante, soit $31.7 \%$ des centres faiblement efficaces avec des scores inférieurs à 0.487 .

La détection des rendements d'échelle variables au niveau des trois classes de centres, nous ramène à estimer la forme REV du modèle BCC(1984) et à analyser ses résultats (ETP) en déduisant les scores de l'efficacité d'échelle (ECH) (Annexes, tableau 1, $2^{\text {ème }}$ étape).

Les scores de l'ECH des établissements varient entre 0.207 et 1. Ainsi, nous avons constaté que les centres du groupe (1) ont réalisé des économies d'échelle dans la mesure où l'ECH dépasse $80 \%$, c'est-à-dire ils ont fonctionné avec des rendements d'échelle constants. Concernant les centres du groupe (2), ils se décomposent en deux sous groupes, le premier renferme $46 \%$ des centres efficaces (0.807-0.899) supposés ayant des rendements d'échelle constants, par contre, le deuxième sous groupe est composé de centres ayant des rendements d'échelle variables (croissants ou décroissants) puisque leurs ECH sont comprises entre 0.548 et 0.781 . Il semble ici que c'est le coût dû à la présence des rendements d'échelle croissants ou décroissants qui devient plus important.

Enfin, les centres du groupe (3) ont tous fonctionné avec des rendements 
d'échelle d'où la présence des inefficacités d'échelle dans la mesure où les scores ECH sont inférieurs à 0.703 .

La variabilité entre les niveaux de performance des groupes s'explique par les caractéristiques des centres. En effet, le taux d'utilisation de la capacité de formation (TUC) indique une sous utilisation de la capacité pour les groupes G3 et $\mathrm{G} 2$ respectivement de $85 \%, 92 \%$ alors le groupe G1 réalise $113 \%$. Ces derniers ont bien profité des avantages de la formation en alternance et à l'apprentissage en vue de réaliser un meilleur remplissage de leurs capacité (TUC), soit 47.8\%, contre $25.9 \%$ et $24 \%$ respectivement pour les groupes (2) et (3).

Le groupe de centre G1 forme plus de techniciens, $78.6 \%$, et qui sont plus demandés sur le marché de l'emploi, que les autres groupes G2 et G3 ayant respectivement les parts de $39 \%$ et $15 \%$. Les taux d'encadrement technique (TET) et administratif (TEA) indiquent un sous-emploi du personnel dans les groupes faiblement et moyennement efficaces. Ces taux sont d'environ 11,6 et 15,2 stagiaires et 15,8 et 22,7 stagiaires respectivement pour le G3 et G2. Le groupe G1 utilise son personnel dans l'ordre de 17,3 stagiaires par formateur et 32,4 stagiaires par administrateur.

Le taux de déperdition (TDEP) est élevé pour tous les groupes. Toutefois, il signale une différence entre les groupes expliquée par le taux d'abandon (TAB) trop élevé pour le G3 par rapport aux G2 et G1. La valeur de la formation paraît nettement faible pour G3 et G2 en comparaison avec celle du G1 ayant respectivement des valeurs moyennes de $59 \%, 77 \%$ et $87,2 \%$.

Pour montrer à quel poids chacune de ces caractéristiques peut influencer le niveau de l'ETG et l'ECH, nous avons expliqué ces deux grandeurs par les variables op-citées suivant une régression linéaire. Les résultats de l'estimation montrent que les variables choisies expliquent bien la variabilité de l'ETG et l'ECH $\left(\mathrm{R}^{2}=0.85\right)$. Ainsi, la valeur de la formation a l'effet le plus élevé aussi bien sur les scores de l'ETG que sur les scores de l'ECH, soit respectivement $0.5562(\mathrm{t}=19.9)$ et $0.504(\mathrm{t}=7.709)$. Puis, on trouve les effets du TET et TEA qui sont positifs et significatifs soit respectivement pour l'ETG ayant les valeurs $0.2052(\mathrm{t}=7.709)$ et $0.0212(\mathrm{t}=2.063)$. Pour l'ECH, ces deux variables ont les effets respectifs de 0.1796 $(\mathrm{t}=5.786)$ et $0.0139(\mathrm{t}=1.116)$.

Les autres variables ont des effets inverses dans la mesure où l'ETG et l'ECH sont respectivement positives et négatives quand le TUC varie alors qu'elles sont négatives et positives quand les établissements appliquent des programmes normalisés (PN).

\section{Analyse des résultats de l'estimation du modèle des coûts d'ombre et déduction de l'EA}

L'estimation des élasticités a été effectuée indirectement ${ }^{6}$ en deux étapes. La première consiste à estimer le modèle des coûts habituels. Les résultats obtenus 
(Annexes, tableau 2) montrent que les variables choisies expliquent bien le modèle et leurs effets estimés sont conformes aux attentes théoriques.

Les élasticités des coûts par rapport aux prix des facteurs variables (wi) montrent que les coûts observés augmentent significativement suite à une augmentation relative du prix de la formation. Ces élasticités sont plus élevées dans le cas d'une formation en mode résidentiel que celle en alternance et d'une formation de techniciens que celle de main d'œuvre qualifiée. En effet, les valeurs de $\mathrm{E}_{\mathrm{c}} / \mathrm{wftr}, \mathrm{E}_{\mathrm{c}} / \mathrm{wftal}, \mathrm{E}_{\mathrm{c}} / \mathrm{wfmor}, \mathrm{E}_{\mathrm{c}} / \mathrm{wfmoal}, \mathrm{E}_{\mathrm{c}} /$ wmoap sont égales respectivement à $0.46 \% \quad(t=12.412),, 0.143 \% \quad(t=3.869), 0.207 \% \quad(t=7.203), 0.068 \% \quad(t=2.528)$, $0.121 \%$.

La sensibilité des coûts par rapport à la valeur des qualifications est positive et hautement significative, $\mathrm{E}_{\mathrm{c}} /$ production $0.7 \%(\mathrm{t}=9.339)$. Cependant cette sensibilité est significativement négative par rapport au facteur travail, $\mathrm{E}_{\mathrm{c}} /$ travail $-1.222(\mathrm{t}=-8.225)$ et nulle par rapport au nombre de postes utilisés. Ceci peut signifier, qu'à court terme, une augmentation de l'effectif du personnel technique améliorera le taux d'encadrement, et par conséquent, la qualité de formation qui se reflètera bien sur le taux de réussite des stagiaires et sur l'insertion des diplômés. C'est-à-dire une augmentation de la valeur créée sans coût marginal supplémentaire ce qui réduit le coût variable total. Par contre, cet effet ne se produit pas par la capacité de formation.

La deuxième étape représente une estimation du modèle des coûts d'ombre. Leurs résultats (Annexes, tableau 3) montrent que les modèles des coûts d'ombre, ACK et MB, s'expliquent bien avec des taux respectifs corrigés $\left(\bar{R}^{2}\right)$ dépassant les $60 \%$. Les paramètres obtenus sont interprétés comme les effets des caractéristiques de la formation (niveau et mode) sur les distorsions des prix de la formation. Il parait, selon l'estimation ACK, que toutes les catégories de formation, sauf celle relative à la formation des CAP en apprentissage, réduisent les distorsions des prix respectifs. Par contre, l'estimation MB révèle que les effets des caractéristiques des catégories de formation des techniciens provoquent une faible réduction non significative des distorsions des prix. Par contre, les effets des catégories de formation des CAP dépendent du mode de formation à savoir l'effet de la formation en modes résidentiel et par l'apprentissage qui sont positifs alors que celui de la formation en alternance est négatif.

Avec le calcul de la distorsion des prix issu des résultats obtenus sur la base d'une analyse financière à court terme, on peut suggérer que les achats publics sont soumis à des procédures d'appel d'offre public dont la nature s'échappe aux mécanismes du marché concurrentiel obéissant à la loi de l'offre et de la demande. Dans la pratique, la gestion financière des centres a été centralisée non seulement au niveau de l'Agence Tunisienne de la formation professionnelle (ATFP) qui gère le dispositif mais aussi au niveau de chaque grande région où il existe un seul économat gérant les dépenses des centres qui lui appartiennent. 
De ce fait, la centralisation de la gestion financière des centres par région renforce un comportement qui se manifeste par le recours au marché du gros et donc les achats ont été faits selon les prix de gros au lieu des prix du marché.

Dans un sens inverse, ces résultats peuvent être expliqués par le comportement des fournisseurs pouvant être satisfaits à vendre avec des prix inférieurs à ceux du marché concurrentiel à condition que la différence soit compensée par une quantité vendue plus élevée.

Les principales origines de la distorsion des prix sont les caractéristiques de la catégorie de formation (niveau et mode) et les caractéristiques des secteurs de formation. La formation dans les secteurs de l'Electricité et Electronique (EE), Tertiaire (T) et les Bâtiments et les Travaux Publics (BTP) s'accompagne d'une distorsion des prix respectivement de $0.33 \%$, de $6.3 \%$ et de $1.3 \%$.

Les Cuirs et Chaussures (CC), la Conduite et la Maintenance des Véhicules et Engins des Travaux Publics et Agricoles (CMVETPA), la Mécanique Générale et la Construction Métallique (MGCM), le Textile et Habillement (TH), le Tourisme et Hôtellerie (TH) représentent des secteurs où la distorsion des prix s'affaiblit, soit respectivement de $-4.1 \%,-4.8 \%,-18.4 \%,-6 \%$ et $-260 \%$.

\section{Déduction de l'efficacité allocative}

Les scores retenus de l'efficacité allocative sont ceux déduits des résultats issus du modèle de $\mathrm{MB}$ et qui sont plus réalistes. Ces scores montrent que les établissements de formation sont allocativement efficaces avec des valeurs toutes supérieures à 0.803 .

\section{CONCLUSION GENERALE}

L'objectif de notre travail consiste à évaluer l'efficacité des établissements publics de la formation professionnelle diplômante en Tunisie. Nous avons utilisé une technique en deux étapes, l'une non paramétrique basée sur la construction et l'analyse d'un enveloppement de données pour estimer l'efficacité technique et ses composantes et l'autre paramétrique reposant sur l'analyse de l'estimation des fonctions des coûts et des coûts d'ombre pour estimer l'efficacité allocative (prix) en utilisant les résultats de la première étape.

Les principaux résultats de la première étape montrent que, selon l'efficacité technique, les centres peuvent être classés en 3 groupes: le premier contient $25 \%$ des centres hautement efficaces, le deuxième regroupe $43 \%$ de centres moyennement efficaces et le reste $(32 \%)$ forme le troisième groupe de centres faiblement efficaces. L'explication de l'efficacité technique montre qu'elle est croissante en fonction de la valeur de la formation sur le marché de l'emploi, du pourcentage de la formation dans l'entreprise. Par contre, elle est décroissante en fonction du volume de la capacité et du nombre d'heures du travail du personnel. Quand aux résultats de 
la deuxième étape ils prouvent que les centres sont tous allocativement efficaces.

Généralement, le nouvel environnement créé par le programme

MANFORM peut inciter à une dynamique au sein du dispositif public de la formation initiale permettant d'orienter son environnement à une concurrence guidée par la recherche de la qualité, la maîtrise des coûts de formation et l'accueil du maximum de stagiaires.

\section{Notes:}

${ }^{1}$ la forme primale du modèle BCC (1984) s'écrit comme un système d'équations:

$$
\begin{aligned}
\operatorname{Min}_{\left(\theta_{k}, \lambda_{i}\right)} \theta_{k} & \\
\text { St: } & \sum y_{r j} \lambda_{j} \geq y_{r k} \quad \mathrm{r}=1, \ldots \ldots . \mathrm{s}, \\
& \theta_{k} x_{i k}-\sum x_{i j} \lambda_{j} \geq 0 \quad \mathrm{i}=1 \ldots ., \mathrm{m} \\
& \sum_{j} \lambda_{j}=1, \text { ou } \succ 1, \text { ou } \prec 1
\end{aligned}
$$

${ }^{2}$ La forme générale du modèle des coûts d'ombre ACK s'écrit sous la forme:

$$
\left\{\begin{array}{c}
\ln \left(C^{o b}\right)=\ln \left(C^{*}\right)+\ln (I T)+\ln (A j) \\
S_{i}=\frac{S_{i}^{*} \rho_{i}^{-1}}{\sum_{k} S_{k}^{*} \rho_{k}^{-1}}, \mathrm{I}=1, \ldots \ldots \mathrm{m}-1
\end{array}\right.
$$

$\mathrm{s}_{\mathrm{i}}$ est la part des couts habituel et $\mathrm{s}_{\mathrm{i}}{ }^{*}$ est la part des coûts d'ombre relative au facteur (i).

${ }^{3}$ La forme générale du modèle de MB prend la forme suivante:

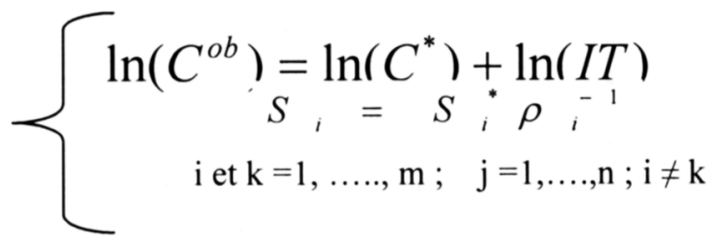

${ }^{4}$ La distorsion des prix de la formation peut être écrite: $r i j=\operatorname{EXP}\left(\mathrm{k}_{\mathrm{i}} * \mathrm{D}_{\mathrm{ij}}+\mathrm{C}_{\mathrm{r}} * \mathrm{R}_{\mathrm{j}}+\mathrm{C}_{\mathrm{s}}\right.$ $* \mathrm{~S}_{\mathrm{j}}$ ). Pour l'estimation, la distorsion sera introduite sous forme de ratio dont celle en dénominateur est choisie arbitrairement, soit celle associée au prix de la formation d'un CAP par l'apprentissage:

$$
\text { rij / r5j }=\operatorname{Exp}(k(i) D i j-k(5) * D 5 j) .
$$


${ }^{5}$ les 5 catégories de stagiaires de formations sont: formation des techniciens (BTS et $\mathrm{BTP}$ ) en résidentiel (ftr), formation des techniciens en alternance (ftal), formation des $\mathrm{CAP}$ en résidentiel (cr), formation des CAP en alternance (cal) et formation des CAP en apprentissage (cap).

${ }^{6}$ L'estimation directe consiste à estimer tous les paramètres en un seul coup en utilisant le modèle des coûts d'ombre. Dans notre cas, cette technique d'estimation a été limitée par un certains nombre de problèmes relatifs à la singularité, au nombre élevé des variables réduisant le degré de liberté, etc. Malgré les corrections possibles qui ont été effectuées, la qualité des résultats reste mauvaise. Ce qui nous ramène à utiliser la technique indirecte.

\section{Références:}

Banker, R.D., Charnes, A., \& Cooper, W.W. (1984). Some models for estimation technical and scale inefficiency in data envelopment analysis. USA: Management Sciences, 30(5), 1078-1092.

Charnes, A., Cooper, W.W., \& Rhodes, E. (1981). Evaluation program and managerial efficiency: An application of data envelopment analysis to program follows through experiment in US public school education. The Institute of Management Sciences, 27(6), 668-696.

Galley, F., \& Meyer, TH. (1998). Transition de la Formation Professionnelle Initiale à la Vie Active. Rapport de base pour l'OCDE.

Joanis, M. (2002). L'Economie de l'éducation: Méthodologie, constats et leçons. Montréal: CERANO, Série Scientifique.

Kumbhakar, S.C. (1990). Production frontiers, panel data, and time-varying technical inefficiency. North Holland: Journal of Economics, 46, 201-211.

Linna, M., \& Hakkinen, U. (1996). Determinants of cost efficiency finnish hospitals: A comparative of DEA and SFA. Finland: National Research and Development Center of Welfare and Health.

Maeitta, O.W. (2000). The decomposition of cost inefficiency into technical and allocative components with panel data of Italian dairy farms. European Review of Agricultural Economics, 27 (4), 473-495.

Maeitta, O.W. (2002). The normalization of the shadow price vector and the estimation of the cost function: A comparative analysis. Napoli: Centro per la Formazione in Economica e Politica Agraria, Universita di Napoli "Federico II". Working Paper $n^{\circ}$ 5. Retrouver le 28 janvier 2008 à partir du home page d'Ornella Wanda Maietta, http:// wpage.unina.it/maeitta/\#List.

Ministère de Développement et de la Coopération Internationale, (2006). Note d'orientation du 11 ${ }^{\text {ème }}$ plan et de la décade (2007-2016). Tunisie: (pas éditeur).

Ministère de l'Education et de le Formation, (2001). Rapport du 10 ${ }^{\text {ème }}$ plan (2002-2006). Tunisie: non publié.

Morlaix S., (2001). Améliorer l'efficacité du système éducatif: réflexion sur la complémentarité entre organisation publique et logique du marché. Présentation

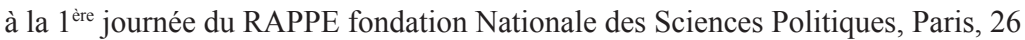


et 27 mars. Retrouver le 24 janvier 2008 à partir du site: www.u-bourgogne.fr/ LABO-IREDU/2001/01001.pdf

Mosbah, E. (2007). Mesure et analyse de l'efficacité du dispositif public de la formation professionnelle en Tunisie dans le cadre de l'application du programme MANFORM. Thèse de doctorat. Tunisie: Institut National Agronomique (INAT).

OCDE (2002). Des innovations dans l'enseignement: Gestion des établissements de nouvelles approches.

Oddie, G. (1966). School building resources and their effective use: Some available techniques and their policy implications. OCDE.

Sall, N. (1996). Les types d'efficacité. Dans Efficacité et équité de l'enseignement supérieur: Quels étudiants réussissent à l'université de Dakar. Tome 1: Cadre théorique et méthodologique (pp.103-116). Thèse de doctorat. Dakar: université cheikh Anta Diop CUSE-FASTET- Faculté des sciences et technologies de l'éducation et de la formation. Retrouver le 24 janvier 2008 à partir du site: http:// www.fastef-portedu.ucad.sn/cuse/cr/cuzon.html 
Annexes

Tableau 1. Synthèse des résultats de l'ETG et ses composantes ETP et ECH

\begin{tabular}{|c|c|c|c|c|c|c|c|c|c|c|}
\hline \multicolumn{5}{|c|}{ 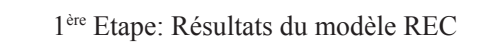 } & \multicolumn{6}{|c|}{$2^{\text {ème }}$ Etape: Résultat du modèle REV } \\
\hline \multicolumn{2}{|c|}{ Indicateurs $^{5}$} & \multirow{2}{*}{\begin{tabular}{|l|} 
Score \\
$0.158-$ \\
1.000
\end{tabular}} & \multirow{2}{*}{\begin{tabular}{|l|}
$\mathrm{NC}$ \\
60
\end{tabular}} & \multirow{2}{*}{$\begin{array}{l}\% \\
100\end{array}$} & \multicolumn{2}{|c|}{ Indicateurs } & \multirow{3}{*}{\begin{tabular}{|l|} 
Scores \\
$0.587-$ \\
1.000 \\
\end{tabular}} & \multirow{2}{*}{$\begin{array}{l}\begin{array}{l}\text { Scores par } \\
\text { sous groupe }\end{array} \\
0.803-1.00\end{array}$} & \multirow{2}{*}{\begin{tabular}{|l|}
$\mathrm{NC}$ \\
33 \\
\end{tabular}} & \multirow{2}{*}{\begin{tabular}{|l|}
$\%$ \\
55 \\
\end{tabular}} \\
\hline \multirow{6}{*}{ ETG } & Pop & & & & \multirow{10}{*}{ ETP } & \multirow[t]{2}{*}{ Pop } & & & & \\
\hline & \multirow[t]{2}{*}{ G1 } & \multirow{2}{*}{$\begin{array}{l}0.807- \\
1.000\end{array}$} & \multirow[t]{2}{*}{15} & \multirow[t]{2}{*}{25} & & & & $\begin{array}{l}0.587- \\
0.798\end{array}$ & 27 & 45 \\
\hline & & & & & & \multirow[t]{2}{*}{ G1 } & \multirow{2}{*}{$\begin{array}{l}0.898- \\
1.000\end{array}$} & - & \multirow[t]{2}{*}{15} & \multirow[t]{2}{*}{100} \\
\hline & \multirow[t]{2}{*}{ G2 } & \multirow{2}{*}{$\begin{array}{l}0.512- \\
0.761\end{array}$} & \multirow[t]{2}{*}{26} & \multirow[t]{2}{*}{43.3} & & & & & & \\
\hline & & & & & & \multirow{3}{*}{ G2 } & \multirow{3}{*}{$\begin{array}{l}0.716- \\
1.000\end{array}$} & $0.803-$ & 13 & 50 \\
\hline & G3 & $\begin{array}{l}0.158- \\
0.487\end{array}$ & 19 & 31.7 & & & & 1.000 & & \\
\hline \multirow{20}{*}{ TRE } & \multirow[t]{4}{*}{ Pop } & REC & 6 & 10 & & & & $\begin{array}{l}0.716- \\
0.798\end{array}$ & 13 & 50 \\
\hline & & RECr & 52 & 86.7 & & & & $0.860-$ & 5 & 26.3 \\
\hline & & RED & 2 & 3.3 & & & 0.938 & & & \\
\hline & & & & & & & & $\begin{array}{l}0.587- \\
0.776\end{array}$ & 14 & 73.4 \\
\hline & G1 & REC & 6 & 40 & & & & $0.807-$ & 27 & 45 \\
\hline & & & & & & Pon & $0.207-$ & & & \\
\hline & & RECr & 7 & 46.7 & & & 1.000 & $0.507-$ & 24 & 40 \\
\hline & & RED & 2 & 13.3 & & & & 0.781 & & \\
\hline & & & & & & & & $0.207-$ & 9 & 15 \\
\hline & G2 & REC & 0 & 0 & ECH & & & 0.490 & & \\
\hline & & & & & & G1 & $0.807-1.00$ & - & 15 & 100 \\
\hline & & RECr & 26 & 100 & & & & & & \\
\hline & & & & & & & & $0.807-$ & 12 & 46.2 \\
\hline & & RED & 0 & 0 & & G2 & $\begin{array}{l}0.548- \\
0.899\end{array}$ & & & \\
\hline & & & & & & & & $0.548-$ & 14 & 53.8 \\
\hline & G3 & REC & 0 & 0 & & & & 0.781 & & \\
\hline & & & & & & & & $0.507-$ & 10 & 52.6 \\
\hline & & RECr & 19 & 100 & & $G_{3}$ & 0207 & 0.703 & & \\
\hline & & RED & 0 & 0 & & & & & & \\
\hline & & & & & & & & $\begin{array}{l}0.207- \\
0.490\end{array}$ & 9 & 47.4 \\
\hline
\end{tabular}

Source: nos estimations

NB: TRE : Type des Rendements d'Echelle; REC : Rendement d'échelle Constant, RECr: rendement d'échelle croissant; RED: rendement d'échelle décroissant;

NC: Nombre des Centres; \%: Pourcentage. 
Tableau 2. Résultats d'estimation du modèle des coûts habituels

\begin{tabular}{|c|c|c|c|c|c|}
\hline \multicolumn{2}{|l|}{ Paramètres } & \multirow{2}{*}{$\begin{array}{c}\text { Coefficient } \\
3.216103\end{array}$} & \multirow{2}{*}{$\begin{array}{r}\text { Std. Error } \\
0.987156\end{array}$} & \multirow{2}{*}{$\begin{array}{r}\text { t-Statistic } \\
3.257948\end{array}$} & \multirow{2}{*}{$\begin{array}{l}\text { Prob. } \\
0.0013\end{array}$} \\
\hline Terme constant & $\mathrm{M}(1)$ & & & & \\
\hline Elasticité coût/wtr & $\mathrm{A}(1)$ & 0.460872 & 0.037132 & 12.41162 & 0.0000 \\
\hline Elasticité coût/wtal & $\mathrm{A}(2)$ & 0.143100 & 0.036984 & 3.869267 & 0.0001 \\
\hline Elasticité coût/wcr & $\mathrm{A}(3)$ & 0.206819 & 0.028712 & 7.203162 & 0.0000 \\
\hline Elasticité coût/wcal & $\mathrm{A}(4)$ & 0.068451 & 0.027077 & 2.528034 & 0.0120 \\
\hline Elast. Double c/ wtr*wtr & $\mathrm{A}(11)$ & -0.011117 & 0.001219 & -9.121455 & 0.0000 \\
\hline Elast. Croisée c/ wtr*wtal & $\mathrm{A}(12)$ & -0.001827 & 0.000806 & -2.266537 & 0.0242 \\
\hline Elast. Croisée c/ wtr*wcr & $\mathrm{A}(13)$ & -0.007706 & 0.000947 & -8.138593 & 0.0000 \\
\hline Elast. Croisée c/ wtr*wcal & $\mathrm{A}(14)$ & -0.000374 & 0.001817 & -0.205576 & 0.8373 \\
\hline Elast. Double c/ wtal*wtal & $\mathrm{A}(22)$ & 0.003918 & 0.002380 & 1.646198 & 0.1009 \\
\hline Elast. Croisée c/ wtal*wcr & $\mathrm{A}(23)$ & 0.006188 & 0.001980 & 3.124479 & 0.0020 \\
\hline Elast. Croisée c/ wtal*wcal & $\mathrm{A}(24)$ & -0.001309 & 0.001150 & -1.138381 & 0.2560 \\
\hline Elast. Double c/ wcr*wcr & $\mathrm{A}(33)$ & -0.001767 & 0.000959 & -1.842778 & 0.0665 \\
\hline Elast. Croisée c/ wcr*wcal & $\mathrm{A}(34)$ & 0.002019 & 0.000752 & 2.686069 & 0.0077 \\
\hline Elast. Double c/ wcal*wcal & $\mathrm{A}(44)$ & 0.000719 & 0.001083 & 0.663973 & 0.5073 \\
\hline Elast. Coût / production & $\mathrm{B}(1)$ & 0.702120 & 0.075179 & 9.339330 & 0.0000 \\
\hline Elast. Double c/ production & $\mathrm{B}(2)$ & -0.007425 & 0.003554 & -2.089336 & 0.0376 \\
\hline Elast. Croisée c/ wtr*prod & $\mathrm{G}(1)$ & 0.002244 & 0.000964 & 2.326350 & 0.0207 \\
\hline Elast. Croisée c/ wtal*prod & $\mathrm{G}(2)$ & 0.015263 & 0.003273 & 4.662835 & 0.0000 \\
\hline Elast. Croisée c/ wcr*prod & $\mathrm{G}(3)$ & -0.011581 & 0.002453 & -4.722157 & 0.0000 \\
\hline Elast. Croisée c/ wcal*prod & $\mathrm{G}(4)$ & -0.007152 & 0.002675 & -2.673347 & 0.0080 \\
\hline Elasticité coût-travail & $\mathrm{Z}(1)$ & -1.222647 & 0.148171 & -8.251596 & 0.0000 \\
\hline Elast. double c/cap*cap & $Z(5)$ & 0.059330 & 0.009508 & 6.240079 & 0.0000 \\
\hline Elast. Croisée c/ wtal*travail & $\mathrm{T}(2)$ & -0.099531 & 0.016871 & -5.899553 & 0.0000 \\
\hline Elast. Croisée c/ wcr*travail & $\mathrm{T}(3)$ & 0.041104 & 0.006423 & 6.399181 & 0.0000 \\
\hline Elast. Croisée c/ wcal*travail & $\mathrm{T}(4)$ & 0.049105 & 0.014375 & 3.416128 & 0.0007 \\
\hline Elast. Croisée c/ wtal*capacité & $\mathrm{T}(7)$ & 0.047778 & 0.014781 & 3.232416 & 0.0014 \\
\hline Elast. Croisée c/ wcr*capacité & $\mathrm{T}(8)$ & -0.021400 & 0.005084 & -4.209303 & 0.0000 \\
\hline Elast. Croisée c/ wcal*capacité & $\mathrm{T}(9)$ & -0.026344 & 0.019152 & -1.375493 & 0.1701 \\
\hline Elast. Croisée c/ prod*travail & $\mathrm{H}(1)$ & 0.031684 & 0.005996 & 5.284453 & 0.0000 \\
\hline Elast. Croisée c/ prod*capacité & $\mathrm{H}(2)$ & -0.045059 & 0.006667 & -6.758693 & 0.0000 \\
\hline Elasticité coût-capacité & $Z(2)$ & 0.000 & Ces élastici & ont supposé & nulles \\
\hline Elast. double c/ travail*travail & $\mathrm{Z}(3)$ & 0.000 & suite au test & li montre leu & \\
\hline Elast. Croisée c/ trav* capacité & $\mathrm{Z}(4)$ & 0.000 & non significa & ités au mom & t de \\
\hline Elast. Croisée c/ wtr*travail & $\mathrm{T}(1)$ & 0.000 & l'estimation & modèle habi & elle des \\
\hline Elast. Croisée c/ wtr*capacité & $\mathrm{T}(6)$ & 0.000 & couts. & & \\
\hline
\end{tabular}

Equation 1: $R^{2}=0.81$ et $\overline{R^{2}}=0.62$; Equation 2: $R^{2}=0.91$ et $\overline{R^{2}}=0.85$;

Equation 3: $R^{2}=0.95$ et $\overline{R^{2}}=0.92$; Equation $4: R^{2}=0.92$ et $\overline{R^{2}}=0.87$;

Equation $5: R^{2}=0.99$ et $\overline{R^{2}}=0.99$

Source: nos estimations 
Tableau 3. Résultats d'estimation des modèles des coûts d'ombre

a- Modèle des coûts d'ombre d'Atkinson \& Cornwell (1994) et Kumbhakar (1996)
$\begin{array}{lcccr} & \text { Coefficient } & \text { Std. Error } & \text { t-Statistic } & \text { Prob } \\ \mathrm{K}(1) & -0.673254 & 0.166746 & -4.037590 & 0.0001 \\ \mathrm{~K}(2) & -0.671911 & 0.203459 & -3.302442 & 0.0011 \\ \mathrm{~K}(3) & -0.260190 & 0.141570 & -1.837886 & 0.0671 \\ \mathrm{~K}(4) & -0.196332 & 0.156657 & -1.253263 & 0.2111 \\ \mathrm{~K}(5) & 0.782474 & 0.349137 & 2.241166 & 0.0258 \\ R^{2}=0.81 & & & R^{2}=0.62\end{array}$

b- Modèle des coûts d'ombre de Mensah (1994) et Balk (1997)

$\begin{array}{lrrrr} & \text { Coefficient } & \text { Std. Error } & \text { t-Statistic } & \text { Prob } \\ \mathrm{K}(1) & -0.040355 & 0.030365 & -1.328989 & 0.1849 \\ \mathrm{~K}(2) & -0.069686 & 0.044875 & -1.552914 & 0.1215 \\ \mathrm{~K}(3) & 0.346327 & 0.032961 & 10.50724 & 0.0000 \\ \mathrm{~K}(4) & -0.436236 & 0.072839 & -5.989017 & 0.0000 \\ \mathrm{~K}(5) & 0.767081 & 0.172215 & 4.454217 & 0.0000 \\ R^{2}=0.68 & & & R^{2}=0.66\end{array}$

Source: nos estimations

\section{Ezzeddine Mosbah}

Docteur, Fonctionnaire à l'Observatoire National des Qualifications et des Métiers Ministère de l'Education et de la Formation. Tunisie.

Tel: (216) 71.568.768 Email adresse: ezzeddine_m@yahoo.fr

\section{Mohamed S. Bachta}

Professeur à l'Institut National Agronomique de Tunisie (INAT). Tunisie.

Tel: (216) 71. 287.110 - 71.289.431 - 71.892.785

Email adresse: bachta.medsalah@inat.agrinet.tn 